Article

\title{
Saturation Detection-Based Blocking Scheme for Transformer Differential Protection
}

\author{
Byung Eun Lee ${ }^{1}$, Jinsik Lee ${ }^{2}$, Sung Ho Won ${ }^{3}$, Byongjun Lee ${ }^{4}$, Peter A. Crossley ${ }^{5}$ \\ and Yong Cheol Kang 6,*
}

1 Wind Energy Grid-Adaptive Technology Research Centre, Chonbuk National University, Chonju 561-756, Korea; E-Mail: mpeclab@jbnu.ac.kr

2 Department of Electrical Engineering and Wind Energy Grid-Adaptive Technology Research Centre, Chonbuk National University, Chonju 561-756, Korea; E-Mail: 2jinshik@jbnu.ac.kr

3 Korea Electrical Contractors Association, \#533-2, Deungchon-2Dong, Gangseo-Ku, Seoul 157-718, Korea; E-Mail: wsungho@keca.or.kr

4 Department of Electrical Engineering, Korea University, Seoul 136-701, Korea;

E-Mail: leeb@korea.ac.kr

5 The School of Electrical \& Electronic Engineering, the University of Manchester, Manchester M13 9PL, UK; E-Mail: p.crossley@manchester.ac.uk

6 Department of Electrical Engineering, Wind Energy Grid-Adaptive Technology Research Centre and Smart Grid Research Centre, Chonbuk National University, Chonju 561-756, Korea

* Author to whom correspondence should be addressed; E-Mail: yckang@jbnu.ac.kr; Tel.: +82-63-270-2391; Fax: +82-63-270-2394.

Received: 26 February 2014; in revised form: 27 June 2014 / Accepted: 10 July 2014 / Published: 18 July 2014

\begin{abstract}
This paper describes a current differential relay for transformer protection that operates in conjunction with a core saturation detection-based blocking algorithm. The differential current for the magnetic inrush or over-excitation has a point of inflection at the start and end of each saturation period of the transformer core. At these instants, discontinuities arise in the first-difference function of the differential current. The second- and third-difference functions convert the points of inflection into pulses, the magnitudes of which are large enough to detect core saturation. The blocking signal is activated if the third-difference of the differential current is larger than the threshold and is maintained for one cycle. In addition, a method to discriminate between transformer saturation and current transformer (CT) saturation is included. The performance of the proposed blocking scheme was compared with that of a conventional harmonic blocking method. The test results
\end{abstract}


indicate that the proposed scheme successfully discriminates internal faults even with CT saturation from the magnetic inrush, over-excitation, and external faults with CT saturation, and can significantly reduce the operating time delay of the relay.

Keywords: saturation detection based blocking; harmonic blocking; current differential relay; transformer protection; operating time delay

\section{Introduction}

Transformer protection relays must be able to differentiate internal faults from all other operating conditions, and current differential relays have been widely used for transformer protection. The relays, however, are prone to malfunctioning during magnetic inrush or over-excitation situations. To prevent malfunctions, the relays adopt restraining or blocking signals that are derived from the current, the voltage, or the flux [1].

Current-derived restraining or blocking methods are based on the harmonic contents in the operating currents [2-5] or wave shape identification [6,7]. Hayward [2] and Mathews [3] used all of the harmonics to restrain the differential relay for a transformer. Sharp and Glassburn [4] introduced the idea of harmonic blocking using the second harmonic. Einvall and Linders [5] introduced a composite restraint function with the second and fifth harmonics. The harmonic-based restraining or blocking methods ensure relay security for the magnetic inrush or over-excitation. However, the methods malfunction for cases with very low harmonic contents in the operating current.

Rockefeller [6] proposed a blocking scheme if successive peaks of the differential current fail to occur at about 7.5-10 ms. Another technique was proposed [7] based on the length of the time intervals when the differential current is near zero. During inrush, the low current intervals are greater than one-quarter cycle and the relay is blocked. For internal faults, the low current intervals are less than one-quarter cycle and the relay operates. However, wave shape recognition techniques fail to identify over-excitation.

For the voltage-derived restraint, the so-called "tripping suppressor" [8] uses a voltage relay to block the differential relay if the voltage is high. However, this method is slower than harmonic restraint devices.

Phadke and Thorp [9] proposed a flux-restrained current differential relay. This relay calculates the rate of change of the flux with respect to the differential current and uses that as a restraint. However, the relay uses the winding current, which is unavailable for a transformer with a delta winding.

Other techniques have been reported that rely on the electro-magnetic equations of a transformer [10-13]. The operating time is very fast, but the voltages of the primary and secondary windings are necessary.

Recently, Saleh et al. [14,15] proposed wavelet-based technologies, and Tripathy et al. [16,17] suggested neural network-based technologies for power transformer protection. Oliveira and Cardoso [18,19] proposed Park's vector-based technologies, and Alencar et al. [20] suggested the differential current gradient vector-based technologies. 
Wavelet-based technologies [14,15] were reported to achieve good accuracy. However, choosing the appropriate wavelet is crucial for successful analysis. In addition, they require long data windows and are vulnerable to noise and unpredicted disturbances. These drawbacks limit their application to relay protection. On the other hand, neural networks $[16,17]$ have been proven to be an efficient tool for classifying and identifying the problems of events. However, these techniques require a large computational burden with large data storage for training or comparing, which causes practical difficulties in implementing these techniques.

Park's vector-based technologies [18,19] used a restraining signal obtained from Park's transform. The differential current gradient vector-based technology [20] used the slope of the differential current in each phase. These techniques successfully discriminate between magnetic inrush and internal fault, particularly for simultaneous inrush and internal fault. However, the performance of these techniques was not validated yet in the case of an internal fault with current transformer (CT) saturation.

A transformer differential relay with a core saturation detection algorithm was suggested [21]. If the magnitude of the third-difference function of the differential current exceeds the threshold, a blocking signal is issued and maintained for one cycle to restrain the operation of a current differential relay. In addition, a method was suggested to discriminate between power transformer saturation and CT saturation. However, the criterion to set the threshold for detecting core saturation using the difference function of the differential current was not addressed.

This paper describes an extensive study of [21], which includes the theoretical background of setting the threshold for core saturation detection. The performance of the proposed relay was verified under various conditions such as the magnetic inrush, an internal fault, over-excitation, and external faults with CT saturation.

\section{Saturation Detection of a Power Transformer Core}

A transformer core saturates during the magnetic inrush or over-excitation. In this case, the exciting current, which is the differential current of a transformer, has a point of inflection at the start and end of each saturation period. The third-difference function of the differential current has a large value at those instants, which can be used to detect core saturation. We will describe how to detect saturation of the transformer core in the case of a magnetic inrush. This will be applied to the over-excitation in a similar manner.

\subsection{Analysis of an Inrush Current}

Figure 1 shows a simplified equivalent circuit of a transformer for the analysis of the inrush current and the piecewise-linearized magnetization curve of the transformer core. The voltage $v(t)$ is given by:

$$
v(t)=R i(t)+\frac{\mathrm{d} \lambda(t)}{\mathrm{d} t}
$$

where $R$ is the winding resistance; and $\lambda(t)$ is the flux linkage.

Let us assume that $v(t)=V_{m} \sin (\omega t+\theta)$ is applied to the transformer at $t=0$, the magnetisation inductance in the unsaturated region is linear, i.e., $L_{\mathrm{m}}=L_{m 1}$, and $\lambda(0)=\lambda_{0}$. Then, $\lambda(t)$ before the core saturates will be given by: 


$$
\lambda(t)=\frac{V_{m}}{Z_{\lambda}} \sin \left(\omega t+\theta-\varphi_{1}\right)+\left[\lambda_{0}-\frac{V_{m}}{Z_{\lambda}} \sin \left(\theta-\varphi_{1}\right)\right] e^{-\frac{t}{\tau_{1}}}
$$

where $\tau_{1}=L_{m 1} / R ; Z_{\lambda}=\sqrt{\left(R / L_{m 1}\right)^{2}+\omega^{2}} ; \varphi_{1}=\tan ^{-1}\left(\omega L_{m 1} / R\right)$; and for convenience, $L_{l}$ is ignored.

Figure 1. Simplified equivalent circuit and magnetisation curve of a transformer in the case of the magnetic inrush: (a) simplified equivalent circuit; and (b) magnetisation curve. $L_{l}$ : leakage inductance; $L_{m}$ : magnetisation inductance; $R$ : winding resistance; $v(t), i(t)$ : voltage and current; $\lambda_{\mathrm{s}}$ : core flux at the saturation point; $i_{\mathrm{s}}$ : magnetising current at the saturation point; $L_{m 1}, L_{m 2}$ : magnetisation inductances in the unsaturated and saturated regions.

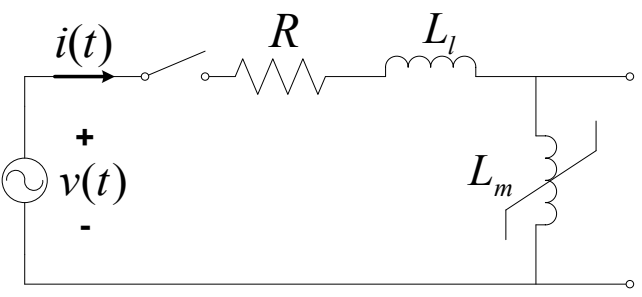

(a)

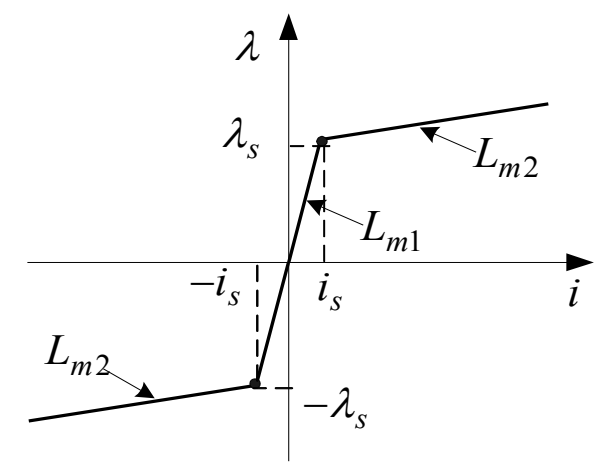

(b)

The current in the unsaturated region $i_{1}(t)$ becomes:

$$
i_{1}(t)=C_{1} \sin \left(\omega t+\theta-\varphi_{1}\right)+\left[\frac{\lambda_{0}}{L_{m 1}}-C_{1} \sin \left(\theta-\varphi_{1}\right)\right] e^{-\frac{t}{\tau_{1}}}
$$

where $Z_{1}=\sqrt{R^{2}+\left(\omega L_{m 1}\right)^{2}}$; and $C_{1}=V_{m} / Z_{1}$.

The current consists of a sinusoid and an exponentially decaying dc component with a time constant $\tau_{1}$. Its discrete-time version is obtained by letting $t=n T$ and $\omega=2 \pi /(N T)$ as follows:

$$
i_{1}[n]=C_{1} \sin \left(\frac{2 \pi}{N} n+\theta-\varphi_{1}\right)+\left[\frac{\lambda_{0}}{L_{m 1}}-C_{1} \sin \left(\theta-\varphi_{1}\right)\right] e^{-\frac{n T}{\tau_{1}}}
$$

where $N$ is the number of samples per cycle; and $T$ is the sampling interval. Defining its first-difference function del1 $[n]$ gives:

$$
\begin{aligned}
\operatorname{del} 1[n]= & i_{1}[n]-i_{1}[n-1] \\
= & \left(2 \sin \frac{\pi}{N}\right) C_{1} \sin \left(\frac{2 \pi}{N} n+\theta-\varphi_{1}-\frac{\pi}{N}+\frac{\pi}{2}\right) \\
& +\left(1-e^{\frac{T}{\tau_{1}}}\right)\left[\frac{\lambda_{0}}{L_{m 1}}-C_{1} \sin \left(\theta-\varphi_{1}\right)\right] e^{-\frac{n T}{\tau_{1}}}
\end{aligned}
$$

As in $i_{1}[n]$, del $1[n]$ consists of a sinusoid and a dc component, but their magnitudes are significantly reduced. First, the reduction rate for a $\operatorname{sinusoid}$ is $2 \sin (\pi / N)$ and depends on $N$. If $N=64$, the magnitude of the sinusoid in $\operatorname{del}\left[1[n]\right.$ is reduced to $2 \sin (\pi / N) C_{1}=0.098 C_{1}$, i.e., a decrease of about $90 \%$. On the other hand, the reduction rate for a dc component is $\left(1-e^{T / \tau 1}\right)$ and depends on $T / \tau_{1}$. If the power system frequency is $60 \mathrm{~Hz}$, then $T=0.26 \mathrm{~ms}$. As $R<<L_{m 1}$ for a transformer, $\tau_{1}>T$. 
Thus, the reduction rate for the exponential term is nearly zero, so the exponential term in $\operatorname{del} 1[n]$ is negligible. Consequently, if $i_{1}[n]$ has a sinusoid and an exponentially decaying dc component, then only the sinusoid in $d e l 1[n]$ exists with a magnitude of $0.098 C_{1}$.

The second- and third-difference functions of $i_{1}[n]$ are defined by:

$$
\begin{aligned}
& \operatorname{del} 2[n]=\operatorname{del} 1[n]-\operatorname{del} 1[n-1] \\
& \operatorname{del} 3[n]=\operatorname{del} 2[n]-\operatorname{del} 2[n-1]
\end{aligned}
$$

where $\operatorname{del} 2[n]$ and $\operatorname{del}[n]$ are sinusoids with magnitudes at $N=64$ of $[2 \sin (\pi / N)]^{2} C_{1} \approx 0.01 C_{1}$ and $(2 \sin (\pi / N))^{3} C_{1} \approx 0.001 C_{1}$, respectively. Hence, the magnitude of the sinusoid in $\operatorname{del} 3[n]$ is reduced to 0.001 of its value in $i_{1}[n]$. Therefore, if $i_{1}[n]$ is in the form of Equation (4), then $\operatorname{del} 3[n]$ is a sinusoid with a magnitude of $0.001 C_{1}$.

Let us assume that the core is saturated at $t=T_{1}$ with $\lambda\left(T_{1}\right)=\lambda_{s}$, where $T_{1}=N_{1} T$, and the magnetisation inductance in the saturated region is $L_{m 2}\left(L_{m 2}<<L_{m 1}\right)$. Then, the current in the saturated region $i_{2}(t)$ can be derived as:

$$
i_{2}(t)=C_{2} \sin \left(\omega t+\theta-\varphi_{2}\right)+\left[\frac{\lambda_{s}}{L_{m 2}}-C_{2} \sin \left(\omega T_{1}+\theta-\varphi_{2}\right)\right] e^{-\frac{t-T_{1}}{\tau_{2}}}
$$

where $\tau_{2}=L_{m 2} / R ; Z_{2}=\sqrt{R^{2}+\left(\omega L_{m 2}\right)^{2}} ; \varphi_{2}=\tan ^{-1}\left(\omega L_{m 2} / R\right)$; and $C_{2}=V_{m} / Z_{2}$.

As in $i_{1}(t)$, the current $i_{2}(t)$ consists of a sinusoid and an exponentially decaying de component with a time constant $\tau_{2}$.

Its discrete-time version, $i_{2}[n]$, becomes:

$$
i_{2}[n]=C_{2} \sin \left(\frac{2 \pi}{N} n+\theta-\varphi_{2}\right)+\left[\frac{\lambda_{s}}{L_{m 2}}-C_{2} \sin \left(\frac{2 \pi}{N} N_{1}+\theta-\varphi_{2}\right)\right] e^{-\frac{\left(n-N_{1}\right) T}{\tau_{2}}}
$$

The magnitude of the sinusoid in $i_{2}[n], C_{2}$, is much larger than $C_{1}$ because $Z_{1}>>Z_{2}$. Its first-difference function for $i_{2}[n]$ can be derived similar to Equation (5). As in Equation (5), the reduction rate for the sinusoid is $2 \sin (\pi / N) \approx 0.1$. As $\tau_{2}>>T$, the reduction rate for a dc component is close to zero and thus the dc component is insignificant in $\operatorname{del}\left[1[n]\right.$. Thus, if $i_{2}[n]$ has the form of Equation (9), then $\operatorname{del} 1[n]$ is a sinusoid with a magnitude of $0.1 C_{2}$.

Similarly, del2[n] and $\operatorname{del} 3[n]$ for $i_{2}[n]$ are sinusoids with magnitudes of $0.01 C_{2}$ and $0.001 C_{2}$, respectively. Hence, the magnitude of the sinusoid in $\operatorname{del} 3[n]$ is reduced to 0.001 of its value in $i_{2}[n]$. Consequently, if $i_{2}[n]$ is in the form of Equation (9), then del3[n] is a sinusoid with a magnitude of $0.001 C_{2}$.

\subsection{Detection of Transformer Core Saturation}

Figure 2 shows a typical inrush current and its difference functions for one cycle, where $V_{m}=140 \mathrm{kV}$, $R=0.94 \Omega, L_{m 1}=33.38 \mathrm{H}$, and $L_{m 2}=0.52 \mathrm{H}$. The inrush current has the forms of Equation (4) (during non-saturation) and Equation (9) (during saturation), respectively. The current has a point of inflection at the start $(n=12)$ and end $(n=50)$ of the saturation period. As shown in Figure $2 \mathrm{~b}$, discontinuities arise at those instants in $\operatorname{del} 1[n]$. These discontinuities are converted into the pulses in $\operatorname{del} 2[n]$ and $\operatorname{del} 3[n]$. Except for those instants, $\operatorname{del} 2[n]$ and $\operatorname{del} 3[n]$ are sinusoids, and their magnitudes are $1 \%$ and $0.1 \%$, 
respectively, of that of the current. The magnitude of the pulse in $\operatorname{del} 3[n]$ is similar to that in $\operatorname{del} 2[n]$, but the magnitude of the sinusoid in $\operatorname{del} 3[n]$ is $10 \%$ of that in $d e l 2[n]$, which can be seen by comparing Figure 2c,d. Thus, the difference between the magnitudes of the pulse and the sinusoid in $\operatorname{del} 3[n]$ is much larger than that in $\operatorname{del}[n]$. Therefore, $\operatorname{del} 3[n]$ is more effective than $\operatorname{del} 2[n]$ for detecting core saturation. In this paper, core saturation is detected if the magnitude of $\operatorname{del} 3[n]$ exceeds the threshold, $T H 1$ :

$$
T H 1=\alpha \sqrt{2} I_{\text {in }{ }_{-} \max }[2 \sin (\pi / N)]^{3}
$$

where $\alpha$ is the constant for the sensitivity of the detection algorithm; and $I_{\text {in } \max }$ is the maximum inrush current. $[2 \sin (\pi / N)]$ is the reduction rate for the sinusoid, and its index, 3 , is the order of the difference-function used for detection. Thus, the terms excluding $\alpha$ in Equation (10) represent the maximum value of the third-difference function of the inrush current.

Figure 2. Inrush current and its difference functions: (a) inrush current; (b) del1 $[n]$; (c) $\operatorname{del} 2[n]$; and (d) $\operatorname{del} 3[n]$.

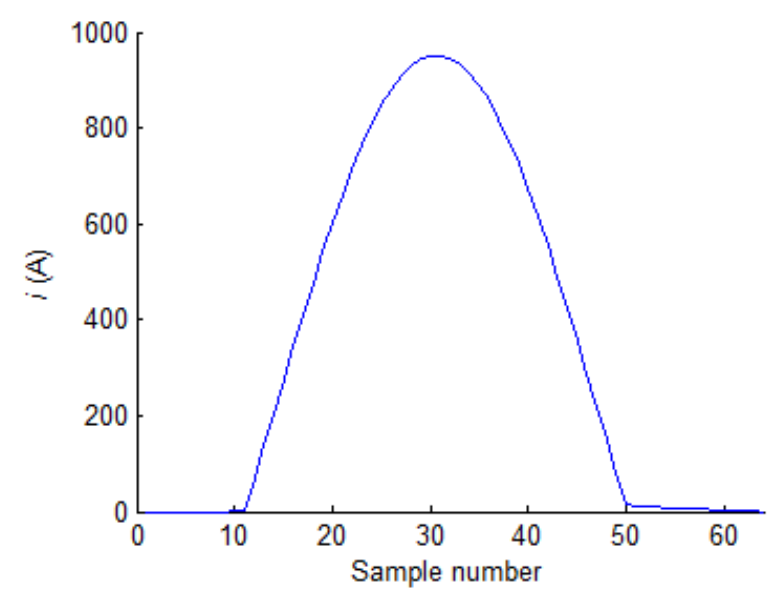

(a)

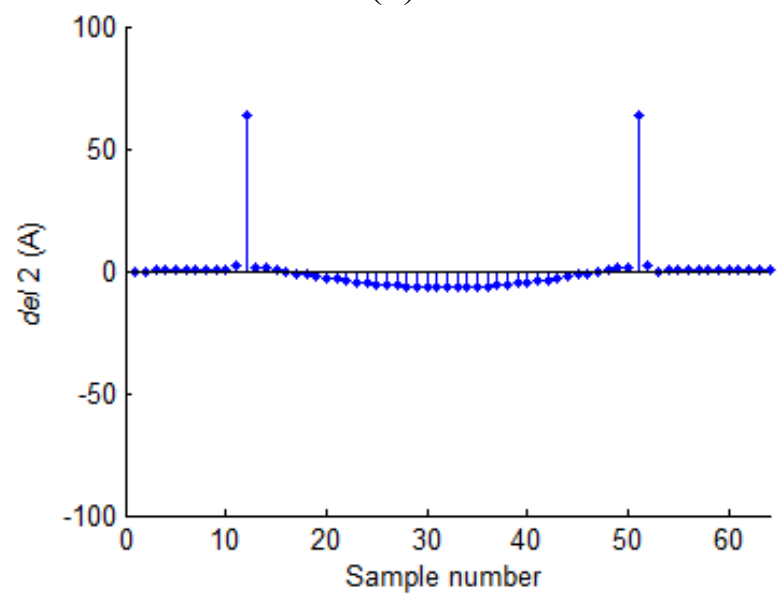

(c)

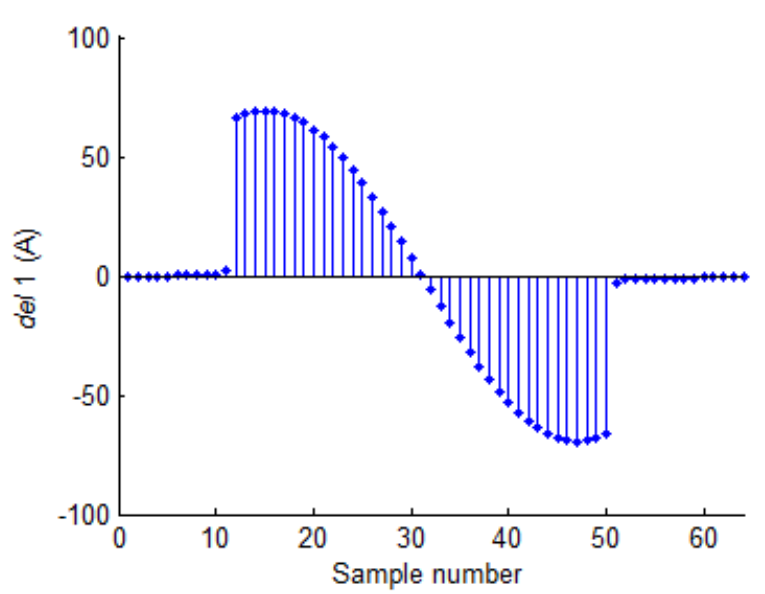

(b)

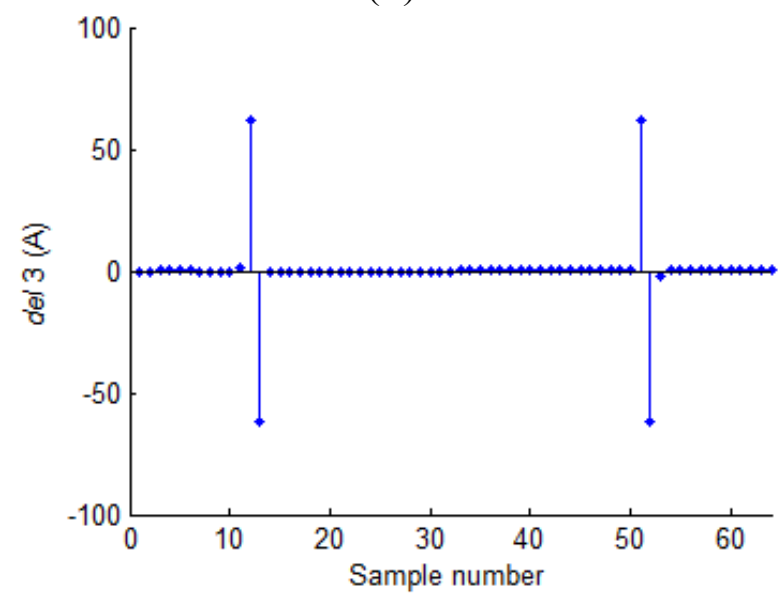

(d)

\section{Current Differential Relay with Core Saturation Detection Blocking}

Figure 3 shows the flowchart of the proposed relay, which consists of two parts, i.e., the current differential relaying protection and the blocking scheme. 
Figure 3. Flowchart of the proposed relaying scheme.

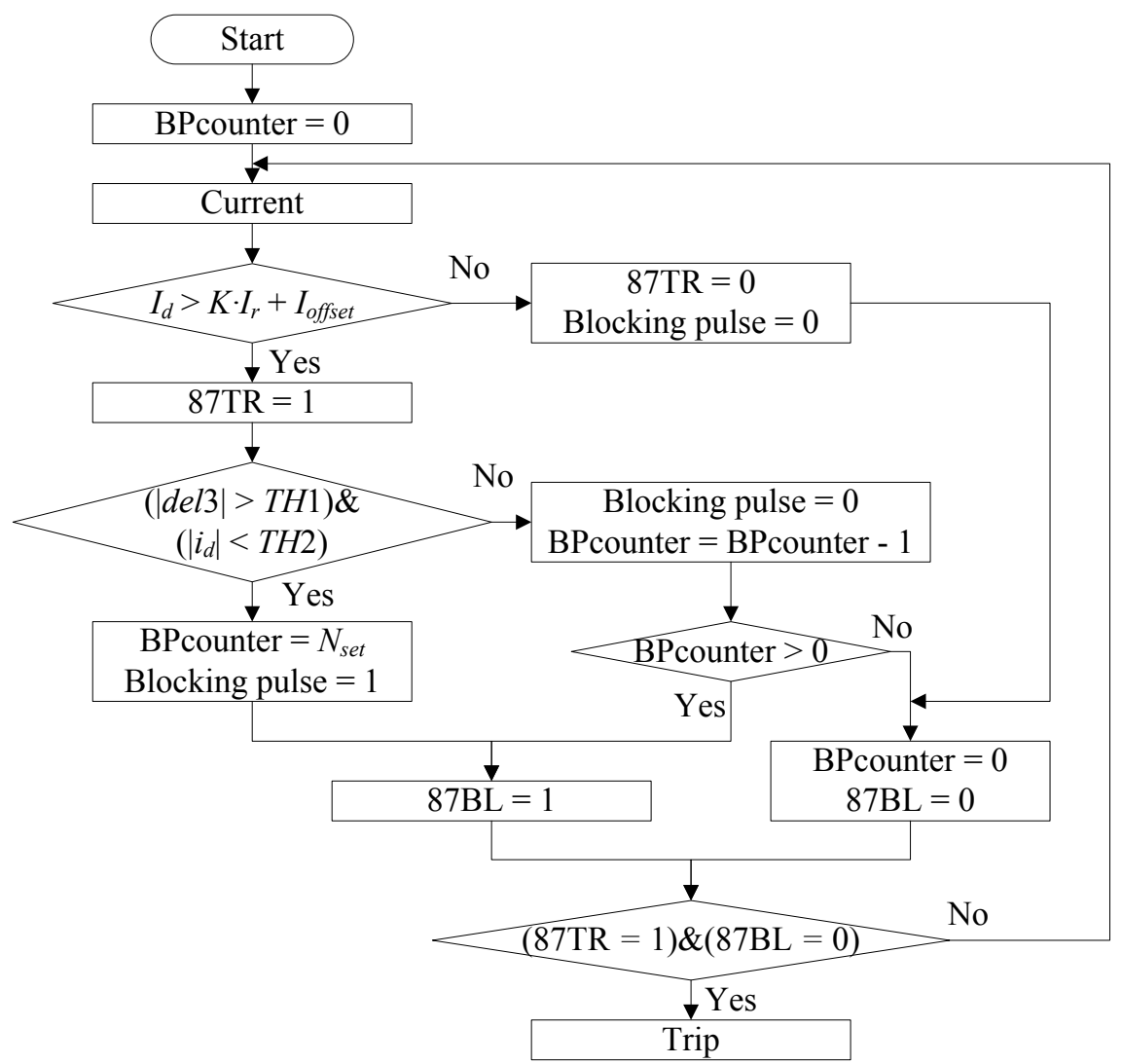

\subsection{Characteristics of a Current Differential Relay}

The differential and restraining currents, $I_{\mathrm{d}}$ and $I_{\mathrm{r}}$, respectively, for the proposed relay in this paper are calculated by:

$$
\begin{gathered}
I_{\mathrm{d}}=\left|\mathbf{I}_{1}-\mathbf{I}_{2}\right| \\
I_{\mathrm{r}}=\left|\mathbf{I}_{1}+\mathbf{I}_{2}\right| / 2
\end{gathered}
$$

where $\boldsymbol{I}_{1}$ and $\boldsymbol{I}_{2}$ are current phasors of the primary and secondary windings of a transformer, referred to the secondary side of the corresponding CTs, respectively.

The characteristic of the differential relay is given by:

$$
I_{\mathrm{d}}>K \cdot I_{\mathrm{r}}+I_{\text {offset }}
$$

where $K=20 \%$; and $I_{\text {offset }}=1 \mathrm{~A}$.

\subsection{Blocking Scheme}

The proposed blocking scheme is based on the following facts: for the magnetic inrush or over-excitation, the differential current has points of inflection repeatedly; whilst for an internal fault, it has a point of inflection only once. At a point of inflection, where the magnitude of $\operatorname{del} 3[n]$ exceeds $T H 1$, a blocking pulse is issued and then the blocking signal (87BL) is activated simultaneously. Once $87 \mathrm{BL}$ is activated, it is maintained for a certain time, i.e., $N_{\text {set, }}$ which is the number of samples to maintain $87 \mathrm{BL}$. 
For an internal fault, the $87 \mathrm{BL}$ is activated at the fault inception and is maintained for one cycle; consequently, the trip signal is activated one cycle after the fault inception. On the contrary, for inrush or over-excitation, where the core repeats saturation and unsaturation, the $87 \mathrm{BL}$ is repeatedly activated and maintained until the core finishes saturation completely.

\subsection{Discrimination between Transformer Saturation and CT Saturation}

If a CT saturates on an internal fault, the differential current also contains points of inflection at the start and end of each saturation period of a CT. In this case, the blocking signal should not be activated. For the magnetic inrush, over-excitation, or an external fault with CT saturation, the differential current contains points of inflection where it has a small value. However, for an internal fault with CT saturation, the differential current has points of inflection where it has a large value. Thus, even though $\operatorname{del} 3[n]$ exceeds $T H 1$, the blocking signal is not activated if the differential current at that instant is larger than the threshold, $T H 2$.

To prevent mis-activation of the blocking signal for an internal fault with CT saturation, a small value of $T H 2$ is desirable. However, if it is too small, the blocking signal may not be activated for the magnetic inrush with CT saturation. In this paper, $5 \mathrm{~A}$, which is the secondary rated current of a CT, is used as $T H 2$.

\section{Case Studies}

Figure 4 shows a single line diagram of the simulated system. A two-winding $\mathrm{Y}-\mathrm{Y}$ transformer (154/22 kV, 55 MVA) was modelled using an electro-magnetic transient program (EMTP) simulator. The currents of the primary and secondary windings were obtained with a sampling rate of 64 samples/cycle $(3840 \mathrm{~Hz})$ and were passed through Butterworth second order filters with a stop-band cut-off frequency of $1920 \mathrm{~Hz}$ (sampling frequency/2). The modelling techniques described in [22] were used to represent internal winding faults.

Figure 4. A single line diagram of the simulated system.
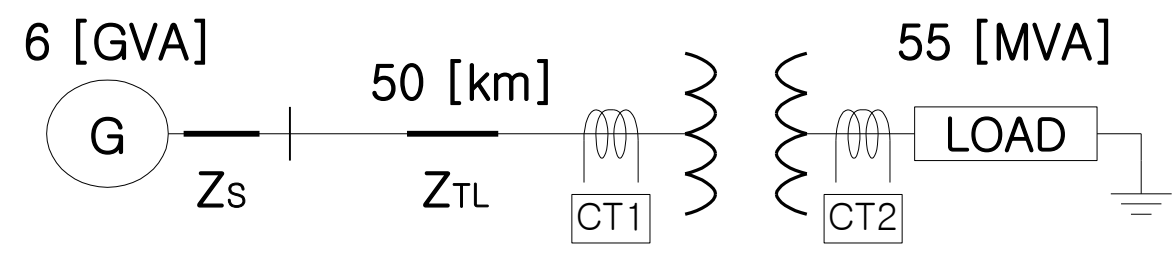

The hysteresis characteristics of the transformer core were modelled using a type-96 element, and a saturation point of $(10 \mathrm{~A}, 334 \mathrm{~V} \cdot \mathrm{s})$ was selected to obtain the hysteresis data by using HYSDAT, a subroutine in EMTP. In addition, two C400 protection CTs, i.e., CT1 (200:5) and CT2 (1400:5), were modelled for the primary and secondary sides, respectively. The saturation point of $(2.05 \mathrm{~A}, 1.51 \mathrm{~V} \cdot \mathrm{s})$ was used to obtain the hysteresis data for CT1 and CT2 using HYSDAT.

Figure 5 shows the diagram for a conventional differential relay with a harmonic blocking method used for comparison, where $I_{2}$ and $I_{5}$ are the magnitudes of the second and fifth harmonics in the differential current, respectively, and the thresholds $K_{2}$ and $K_{5}$, to prevent mal-function during the magnetic inrush or over-excitation, were set to $1 / 21$ and $1 / 25$, respectively, in this paper. The performance 
of the proposed protection scheme was verified and compared with that of the conventional differential relay shown in Figure 5 under various conditions, such as the magnetic inrush with and without CT saturation, internal turn-to-ground with CT saturation, over-excitation, an external fault with CT saturation, and an internal turn-to-turn fault after an external fault. Both relays have the same operating characteristics but different blocking schemes. TH1 for detection of transformer core saturation was set to $0.11 \mathrm{~A}$ in this paper, where $\alpha$ is 1.2 and $I_{\text {in_max }}$ is $100 / \sqrt{2} \mathrm{~A}$. The results for the "A" phase are shown for convenience in this paper.

Figure 5. Conventional differential relay with harmonic blocking.

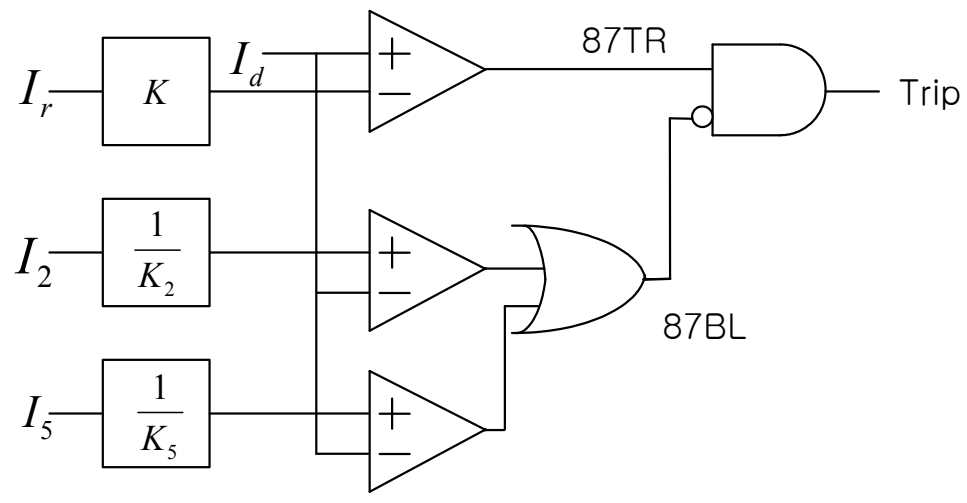

Figures $6 \mathrm{a}-11 \mathrm{a}$ show the instantaneous differential currents, $i_{\mathrm{d}}$, and Figures $6 \mathrm{~b}-11 \mathrm{~b}$ show the loci of $I_{\mathrm{d}}$ and $I_{\mathrm{r}}$. The ratios of $I_{2} / I_{\mathrm{d}}$ and $I_{5} / I_{\mathrm{d}}$ in the differential current are shown in Figures $6 \mathrm{c}-11 \mathrm{c}$. Figures $6 \mathrm{~d}-11 \mathrm{~d}$ show del1[n], del2[n], del3[n], and the blocking pulse. Figures $6 \mathrm{e}-11 \mathrm{e}$ and Figure $6 \mathrm{f}$ show $87 \mathrm{TR}, 87 \mathrm{BL}$, and the trip signals, where the dotted and solid lines indicate the conventional and proposed schemes, respectively.

\subsection{Magnetic Inrush}

Case 1: Energisation angle of $0^{\circ}, 80 \%$ remanent flux in the transformer core, $K_{2}=1 / 5$;

Case 2: Energisation angle of $0^{\circ}, 80 \%$ remanent flux in the transformer core, $K_{2}=1 / 21$.

Figure 6 shows the results for Cases 1 and 2, which are the same except for the value of $K_{2}$. The transformer is energized at $50.1 \mathrm{~ms}$. The large inrush differential current causes the 87TR to enter the operating region at $53.6 \mathrm{~ms}$, i.e., $3.5 \mathrm{~ms}$ after energisation. For the conventional relay, $K_{2}$ is set to the typical value of $1 / 5$ in Case 1 and is set to $1 / 21$ in Case $2 . I_{2} / I_{\mathrm{d}}$ exceeds $K_{2}$ starting at $52.3 \mathrm{~ms}$ in both cases, where the blocking signal $87 \mathrm{BL}$ is activated. However, for Case 1, the blocking signal is deactivated at $62.8 \mathrm{~ms}$ and activated at $103.64 \mathrm{~ms}$ again. Thus, the trip signal is issued at $63.0 \mathrm{~ms}$ for $40.4 \mathrm{~ms}$ as shown in Figure 6e. On the contrary, for Case 2, $K_{2}$ is set to $1 / 21$, which is smaller than that of Case 1. Thus, the conventional relay successfully does not issue the trip signal. For the proposed relay, the first blocking pulse is issued at $52.1 \mathrm{~ms}$ in Cases 1 and 2, where the 87BL is issued at the same time and is maintained for one cycle. Thereafter, the blocking pulses are issued at the start and end of each saturation period, 87BL is successfully maintained, and thus the trip signal is not activated in both cases. 
Figure 6. Results for Cases 1 and 2: (a) $i_{\mathrm{d}}$; (b) loci of $I_{\mathrm{d}}$ and $I_{\mathrm{r}}$; (c) $I_{2} / I_{\mathrm{d}}$ and $I_{5} / I_{\mathrm{d}}$; (d) del1[n], del2[n] and del3[n] of $i_{\mathrm{d}}$, and blocking pulse; (e) $87 \mathrm{TR}, 87 \mathrm{BL}$ and trip signals for Case 1; and (f) $87 \mathrm{TR}, 87 \mathrm{BL}$, and trip signals for Case 2.

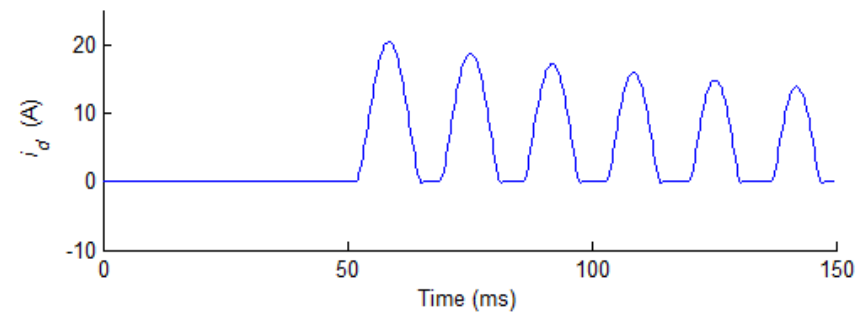

(a)
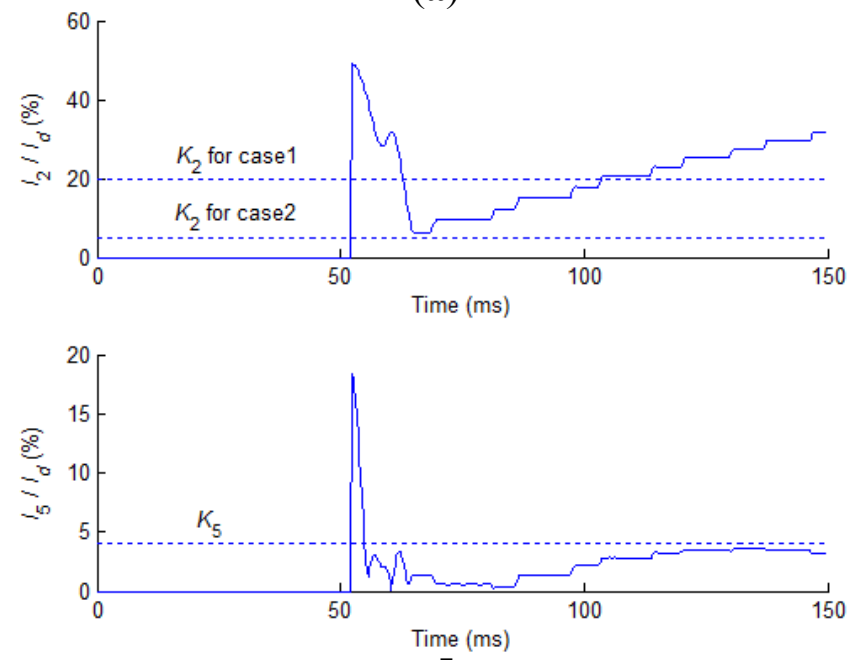

(c)

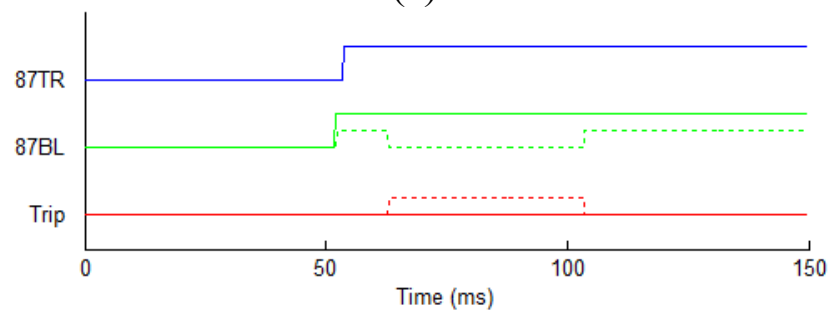

(e)

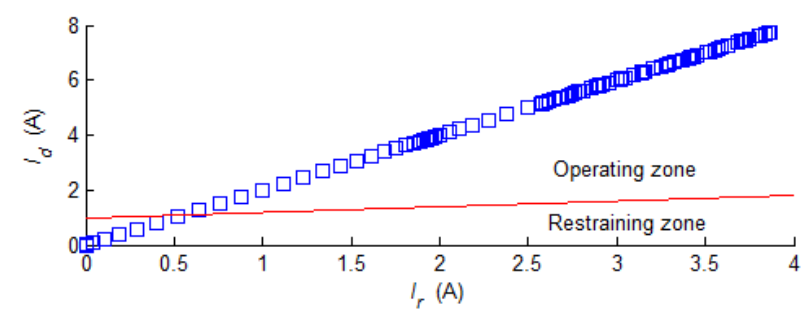

(b)
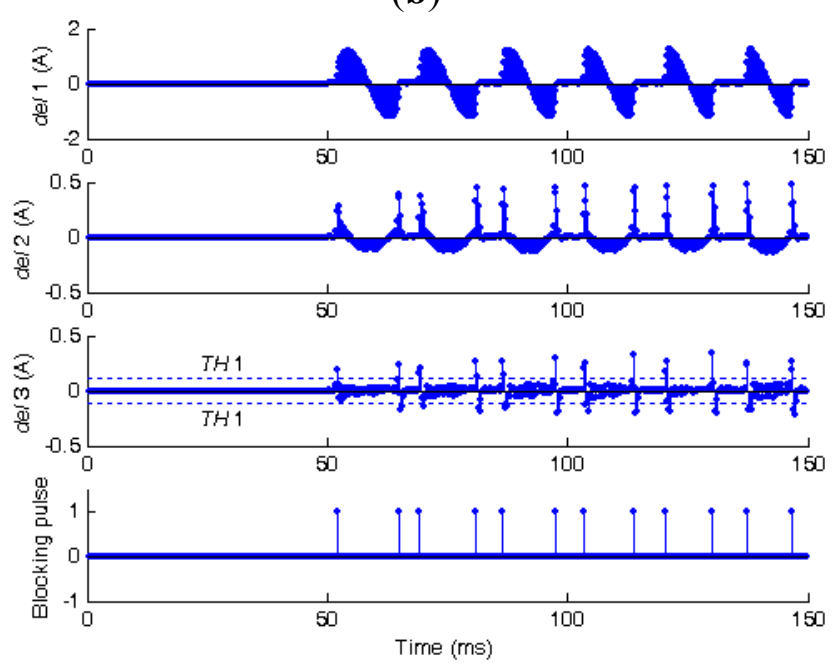

(d)

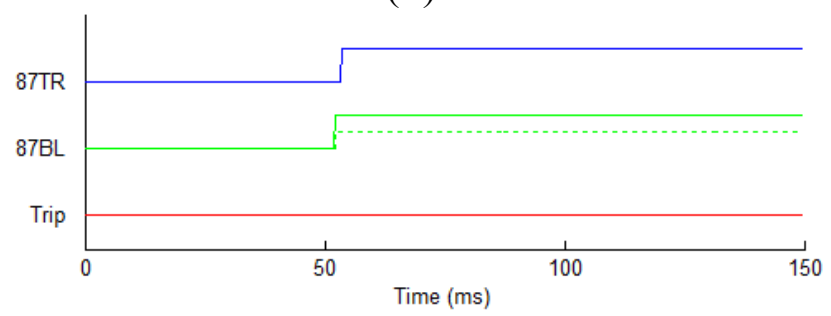

(f)

Case 3: Energisation angle of $0^{\circ}, 80 \%$ remanent flux in the transformer core, and CT saturation.

Figure 7 shows the results for Case 3, which is identical to Case 2 except the CT is saturated. In Figure 7a, the dotted line means the differential current if the CT would not saturate. 87TR enters the operating region at $53.6 \mathrm{~ms}$ similar to Case 2 . The ratio of $I_{2} / I_{\mathrm{d}}$, exceeds $K_{2}$ since $50.3 \mathrm{~ms}$ and the trip signal is not activated. For the proposed relay, the blocking signal is issued at $52.1 \mathrm{~ms}$, where the first blocking pulse is activated, and maintained thereafter. Thus the trip signal is not activated. The results show that both relays enter the operating zone, but both blocking schemes successfully restrain the trip signal for the magnetic inrush with and without CT saturation. 
Figure 7. Results for Case 3: (a) $i_{d}$; (b) locus of $I_{\mathrm{d}}$ and $I_{\mathrm{r}}$; (c) $I_{2} / I_{\mathrm{d}}, I_{5} / I_{\mathrm{d}}$; (d) del1 $[n]$, del2 $[n]$ and $\operatorname{del} 3[n]$ of $i_{\mathrm{d}}$, and blocking pulse; and (e) $87 \mathrm{TR}$, 87BL, and trip signals.

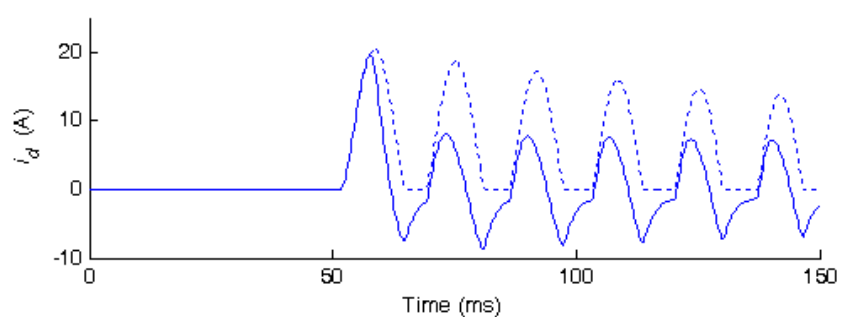

(a)
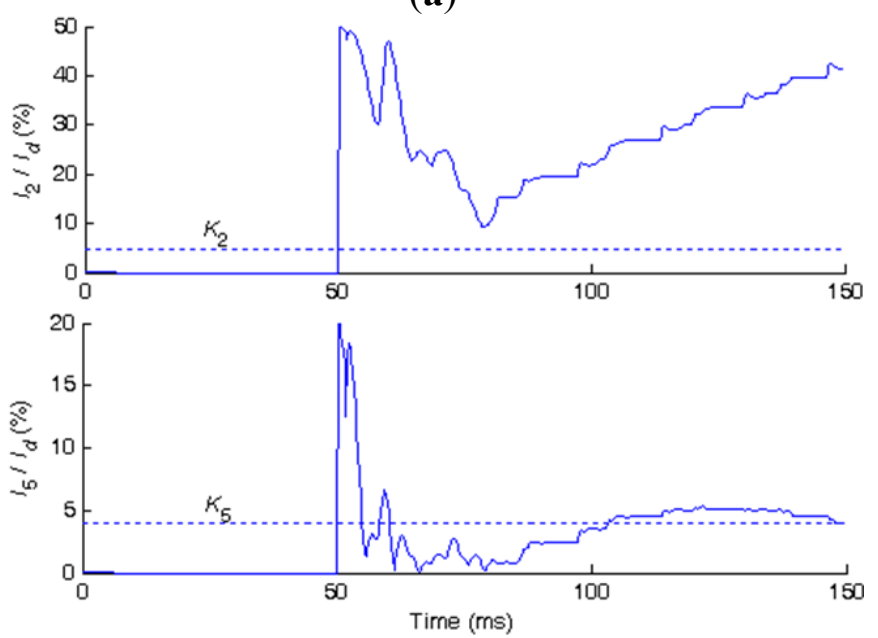

(c)

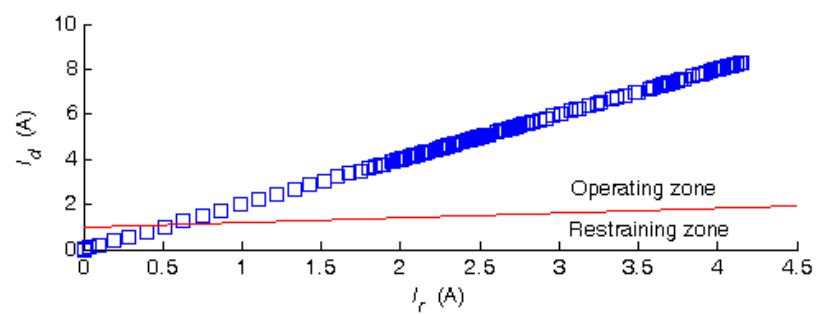

(b)
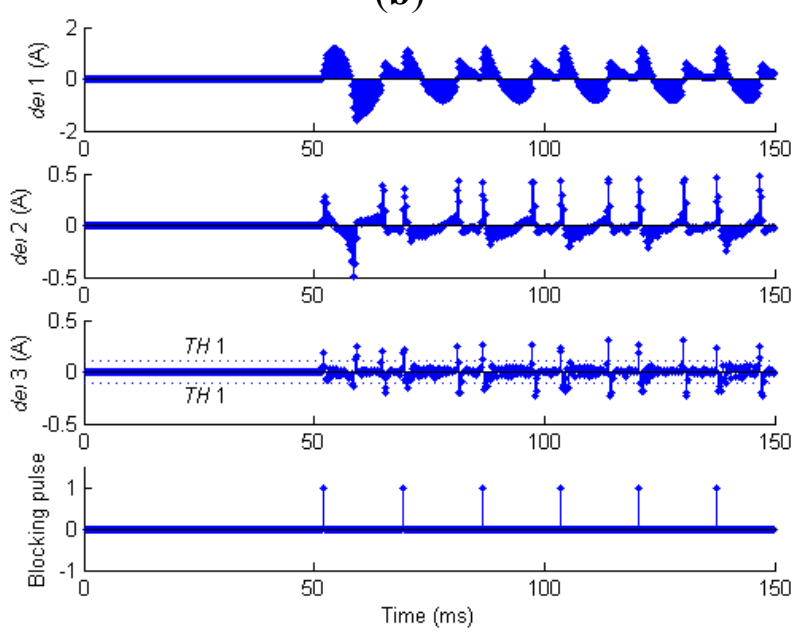

(d)

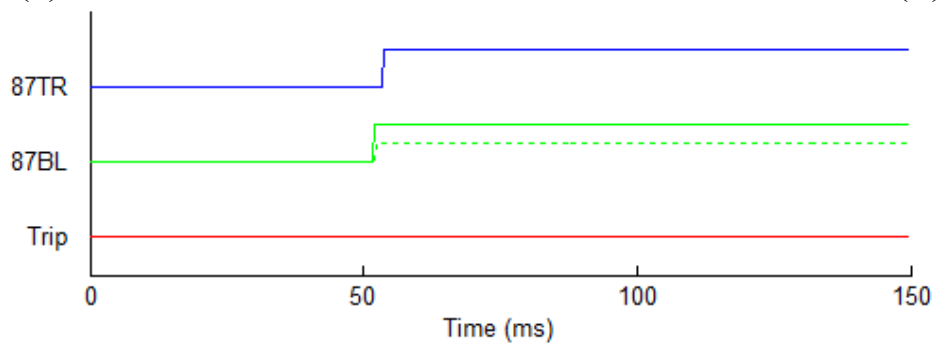

(e)

\subsection{Internal Winding Faults}

Case 4: Turn-to-ground fault, located 50\% from the neutral winding end and at $0^{\circ}$ inception angle, $80 \%$ remanent flux in a CT1.

Figure 8 shows the results for Case 4, where a turn-to-ground fault occurs at $50.1 \mathrm{~ms}$ and an $80 \%$ remanent flux exists in CT1. The differential current is severely distorted due to saturation of CT1 as shown in Figure 8a, where the dotted line indicates the differential current if the CT would not saturate. 87TR enters the operating region at $52.1 \mathrm{~ms}$. The harmonic blocking method is active for the time period of 51.0-128.9 ms. The trip signal is activated at $129.2 \mathrm{~ms}$. The blocking period is significantly large due to the distorted differential current. However, the proposed blocking scheme is active for $50.5-67.4 \mathrm{~ms}$ and the trip signal is issued at $67.7 \mathrm{~ms}$, which is $61.5 \mathrm{~ms}$ earlier than with the harmonic blocking scheme. Thus, the proposed blocking scheme significantly improves the operating speed of a relay when a $\mathrm{CT}$ is saturated for an internal turn-to-ground fault. In this case, we can see some spikes in $\operatorname{del} 3[n]$ after $50.5 \mathrm{~ms}$, such as at $54.4 \mathrm{~ms}, 65.9 \mathrm{~ms}, 72.9 \mathrm{~ms}$, and so on. These spikes are caused by 
CT saturation. As mentioned in subsection 3.3, the blocking pulse is not triggered at these instants because the differential currents at the instants are larger than $T H 2$.

Figure 8. Results for Case 4: (a) $i_{\mathrm{d}}$; (b) loci of $I_{\mathrm{d}}$ and $I_{\mathrm{r}}$; (c) $I_{2} / I_{\mathrm{d}}$ and $I_{5} / I_{\mathrm{d}}$; (d) del1[n], $\operatorname{del} 2[n], \operatorname{del} 3[n]$ of $i_{d}$, and blocking pulse; and (e) $87 \mathrm{TR}, 87 \mathrm{BL}$, and trip signals.

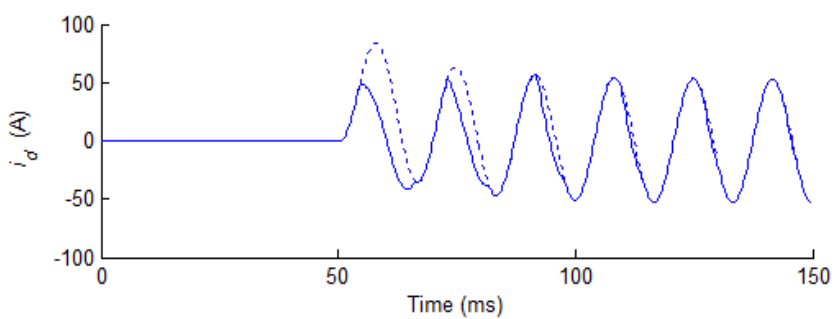

(a)
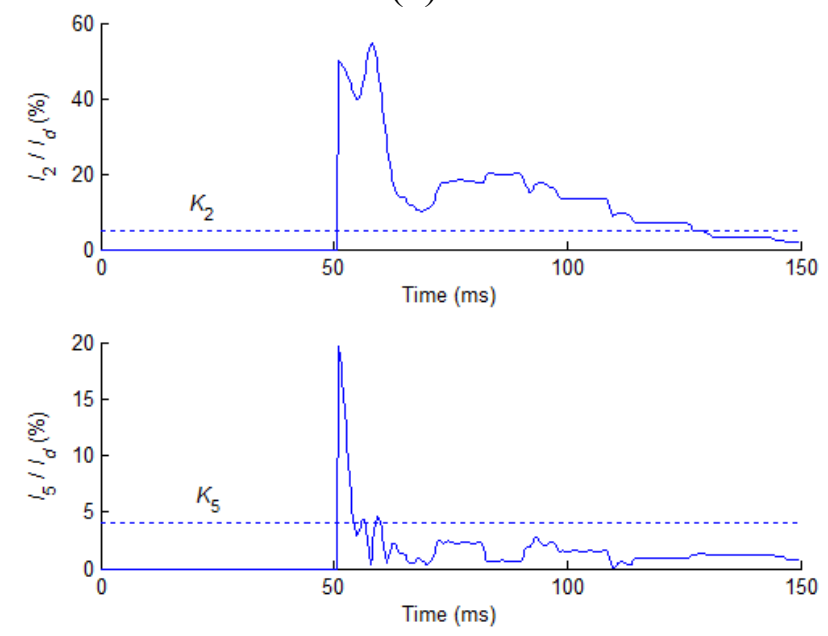

(c)

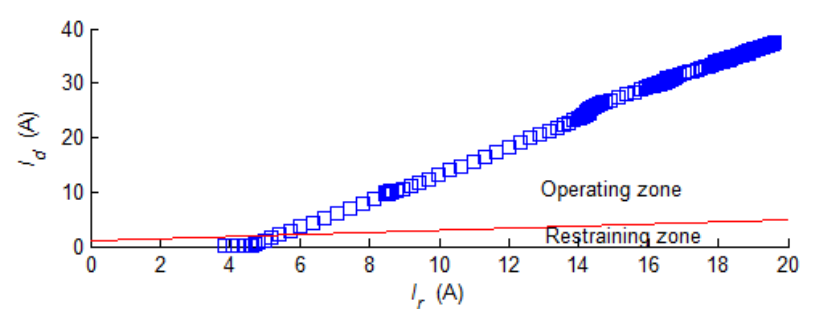

(b)
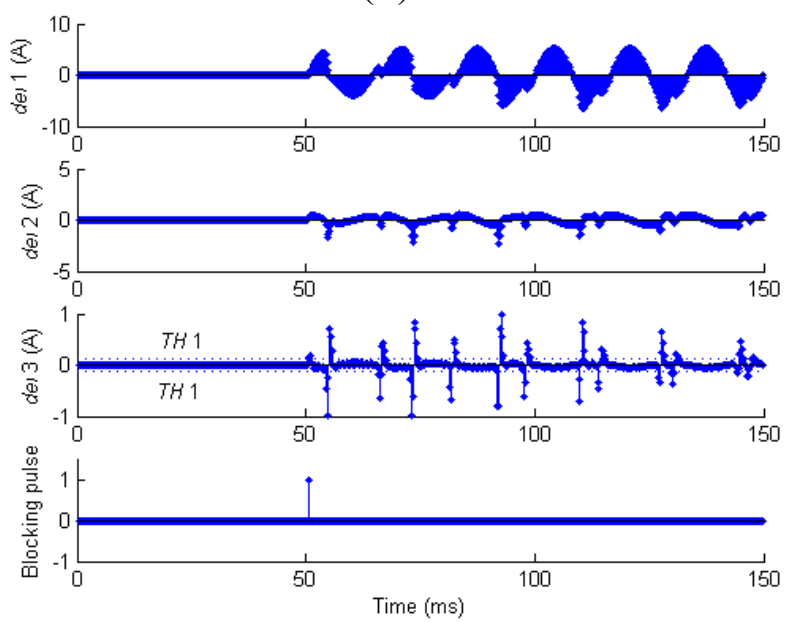

(d)

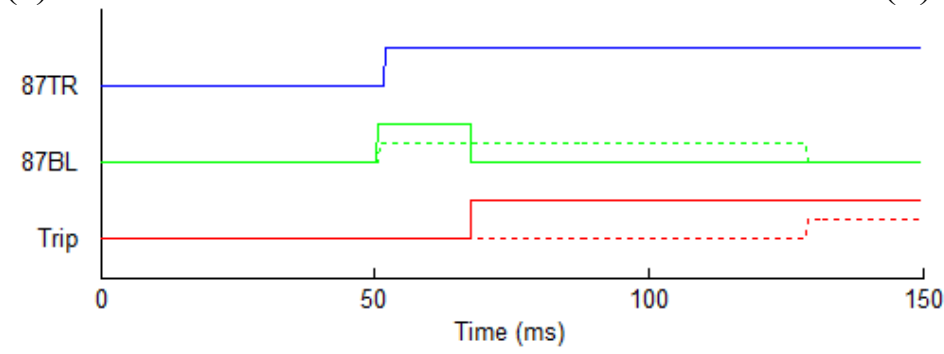

(e)

\subsection{Over-Excitation}

Case 5: 150\% over-excitation.

Figure 9 shows the results obtained when the transformer was over-excited by applying $150 \%$ of the rated voltage at $54.3 \mathrm{~ms}$. The magnitude of the conventional $i_{d}$ is significant as shown in Figure 9a. Since $I_{2} / I_{\mathrm{d}}$ and $I_{5} / I_{\mathrm{d}}$ exceed $K_{2}$ and $K_{5}$, respectively, the blocking signal is issued at $61.4 \mathrm{~ms}$. Meanwhile, the proposed blocking scheme issues the blocking signal at $61.2 \mathrm{~ms}$. Both blocking schemes successfully restrain the trip signal for over-excitation. 
Figure 9. Results for Case 5: (a) $i_{\mathrm{d}}$; (b) loci of $I_{\mathrm{d}}$ and $I_{\mathrm{r}} ;$ (c) $I_{2} / I_{\mathrm{d}}$, and $I_{5} / I_{\mathrm{d}} ;$ (d) dell $[n]$, $\operatorname{del} 2[n]$ and $\operatorname{del} 3[n]$ of $i_{d}$, and blocking pulse; and (e) 87TR, 87BL, and trip signals.

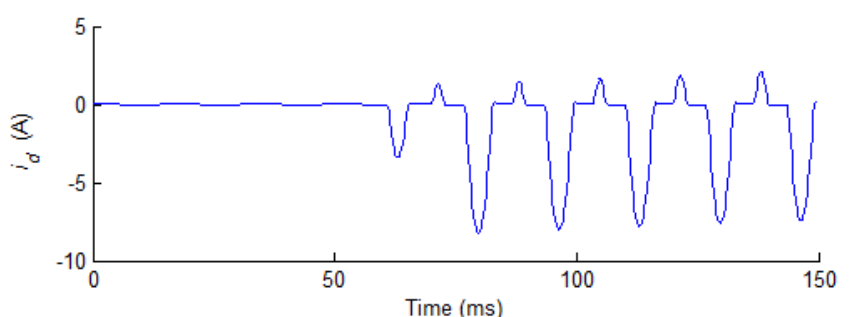

(a)
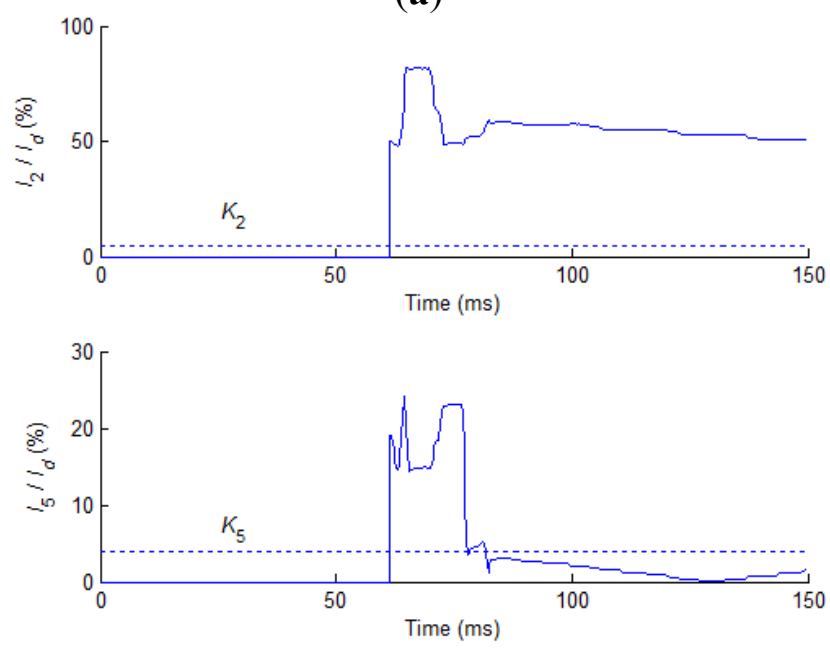

(c)

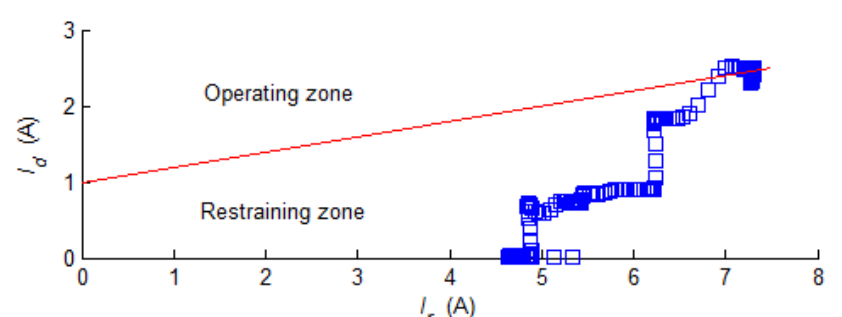

(b)
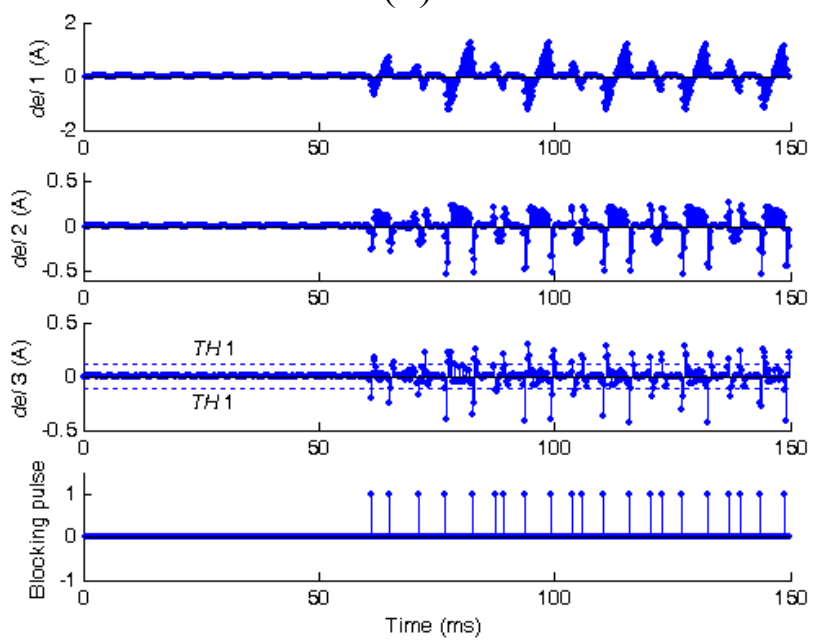

(d)

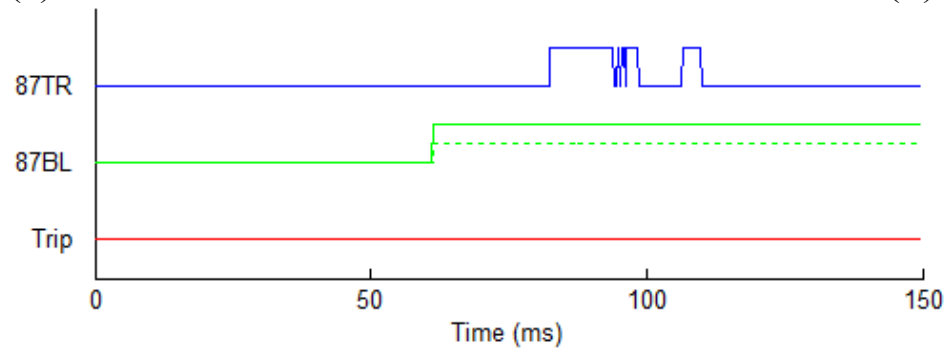

(e)

\subsection{External Fault}

Case 6: External fault, $0^{\circ}$ inception angle, $80 \%$ remanent flux in a CT1.

Figure 10 shows the results for Case 6, where an external fault occurs at $50.1 \mathrm{~ms}$ located outside of the secondary transformer winding. In this case, the CT1 saturates due to the $80 \%$ remanent flux and thus the magnitude of $i_{\mathrm{d}}$ is significant. 87TR enters the operating region at $57.0 \mathrm{~ms}$ and leaves at $81.0 \mathrm{~ms}$. As in the previous cases, $I_{2} / I_{\mathrm{d}}$ and $I_{5} / I_{\mathrm{d}}$ exceed $K_{2}$ and $K_{5}$, respectively, and the harmonic blocking scheme is active for 55.7-97.4 ms. On the contrary, in this case, the blocking pulses at $55.2 \mathrm{ms,}$ $66.4 \mathrm{~ms}$, and $72.1 \mathrm{~ms}$ in Figure 10d are caused by CT saturation. However, unlike Case 4, the differential currents at those instants are less than $T H 2$, and thus the blocking pulses are triggered in this case. The proposed blocking signal is activated during the period of 55.2-89.1 ms, so both blocking schemes successfully restrain the trip signals. 
Figure 10. Results for Case 6: (a) $i_{\mathrm{d}}$; (b) loci of $I_{\mathrm{d}}$ and $I_{\mathrm{r}}$; (c) $I_{2} / I_{\mathrm{d}}$ and $I_{5} / I_{\mathrm{d}} ;$ (d) del1[n], $\operatorname{del}\left[2[n]\right.$ and $\operatorname{del} 3[n]$ of $i_{\mathrm{d}}$, and blocking pulse; and (e) $87 \mathrm{TR}, 87 \mathrm{BL}$, and trip signals.

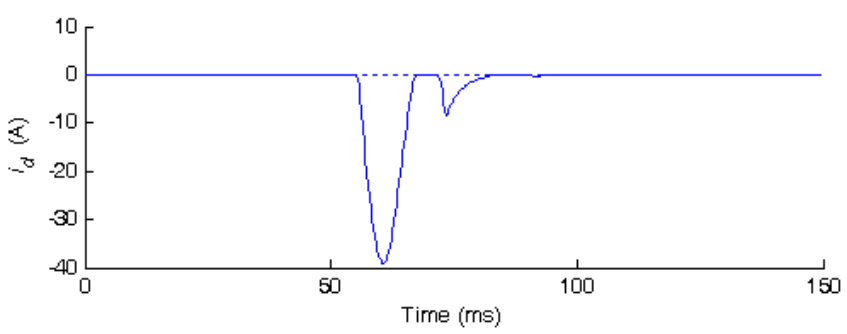

(a)
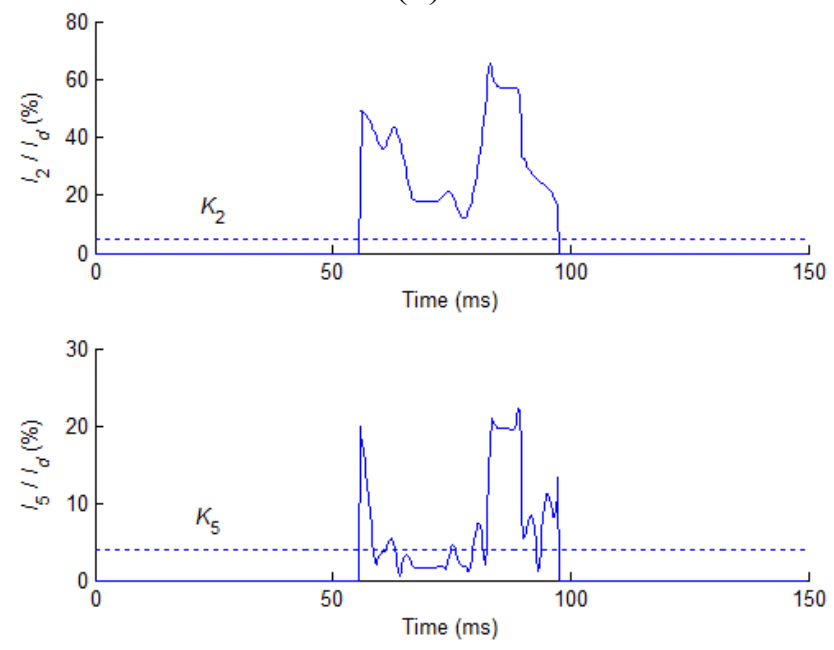

(c)

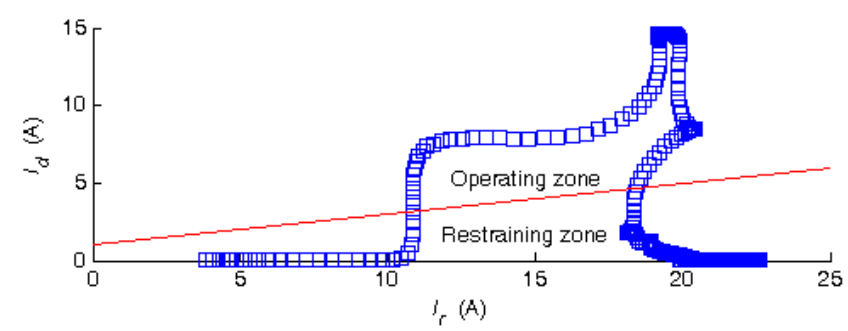

(b)
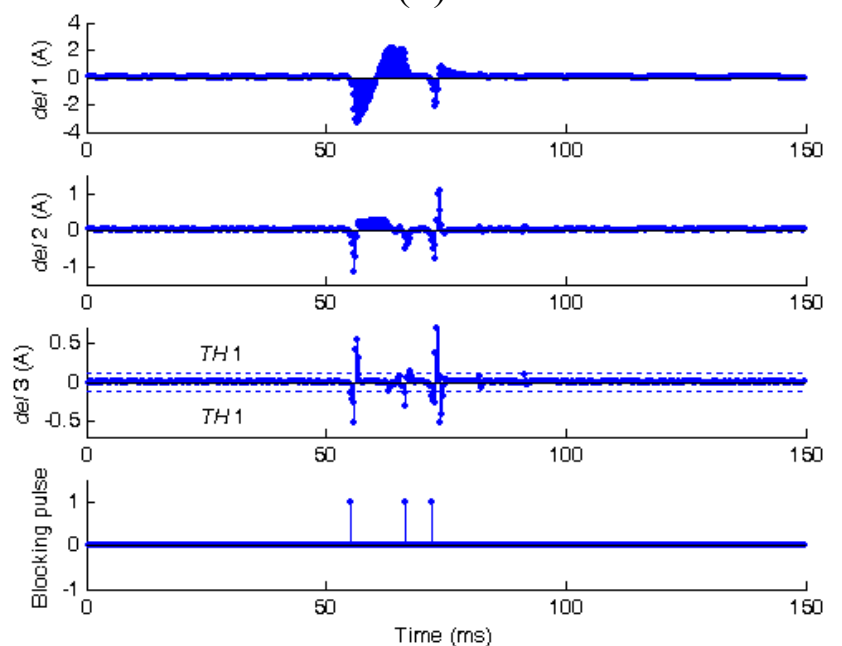

(d)

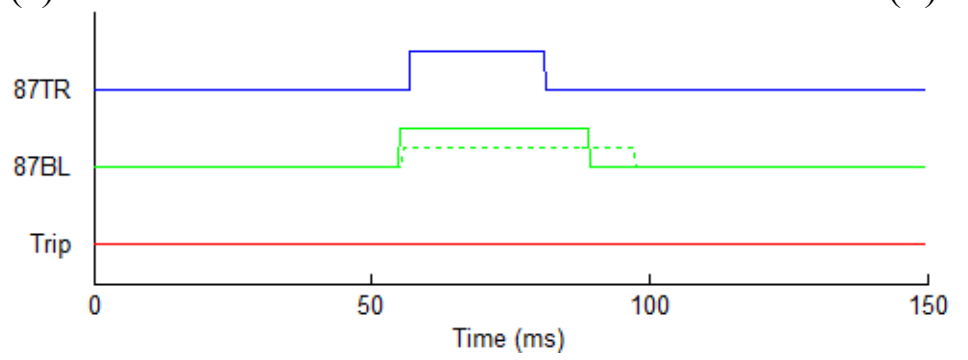

(e)

\subsection{External and Internal Faults}

Case 7: External and turn-to-turn faults, located between $20 \%$ and $10 \%$, and at $0^{\circ}$ inception angle, $80 \%$ remanent flux in a CT1.

Figure 11 shows the results for Case 7, where external and internal faults with $0^{\circ}$ inception angle occur at $50.1 \mathrm{~ms}$ and $66.8 \mathrm{~ms}$, respectively. The external and internal faults are located outside of the secondary transformer winding and between $20 \%$ and $10 \%$ from the neutral winding end, respectively. In this case, the CT1 saturates due to the $80 \%$ remanent flux and thus the magnitude of $i_{\mathrm{d}}$ is significant. 87TR enters the operating region at $57.0 \mathrm{~ms}$. As in Case $4, I_{2} / I_{\mathrm{d}}$ and $I_{5} / I_{\mathrm{d}}$ exceed $K_{2}$ and $K_{5}$, respectively, and the harmonic blocking scheme is active for 55.7-126.6 ms during the external and internal faults. The trip signal is activated at $126.8 \mathrm{~ms}$. However, the proposed scheme activates blocking pulses at $55.5 \mathrm{~ms}$ and $66.4 \mathrm{~ms}$, where the differential currents are less than TH2 (see Figure 11d). After an internal fault, we can see some spikes in $\operatorname{del} 3[n]$, such as at $70.6 \mathrm{~ms}, 81.5 \mathrm{~ms}, 91.4 \mathrm{~ms}$, and so on. These spikes 
are caused by CT saturation. As mentioned in Subsection 3.3, the blocking pulse is not triggered at these instants, where the differential currents are larger than $T H 2$. Thus, the proposed blocking signal is activated for $55.2-83.3 \mathrm{~ms}$. The trip signal is issued at $83.6 \mathrm{~ms}$ during the internal fault, which is $43.5 \mathrm{~ms}$ earlier than the conventional harmonic blocking scheme. Thus, the proposed blocking scheme significantly improves the operating speed of a relay for external and internal faults.

Figure 11. Results for Case 7: (a) $i_{\mathrm{d}}$; (b) loci of $I_{\mathrm{d}}$ and $I_{\mathrm{r}}$; (c) $I_{2} / I_{\mathrm{d}}$ and $I_{5} / I_{\mathrm{d}}$; (d) $\operatorname{del} 1[n]$, $\operatorname{del} 2[n]$ and $\operatorname{del} 3[n]$ of $i_{\mathrm{d}}$, and blocking pulse; (e) $87 \mathrm{TR}, 87 \mathrm{BL}$, and trip signals.

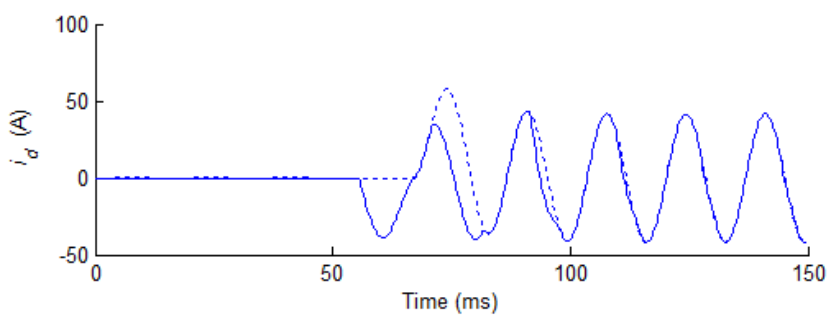

(a)
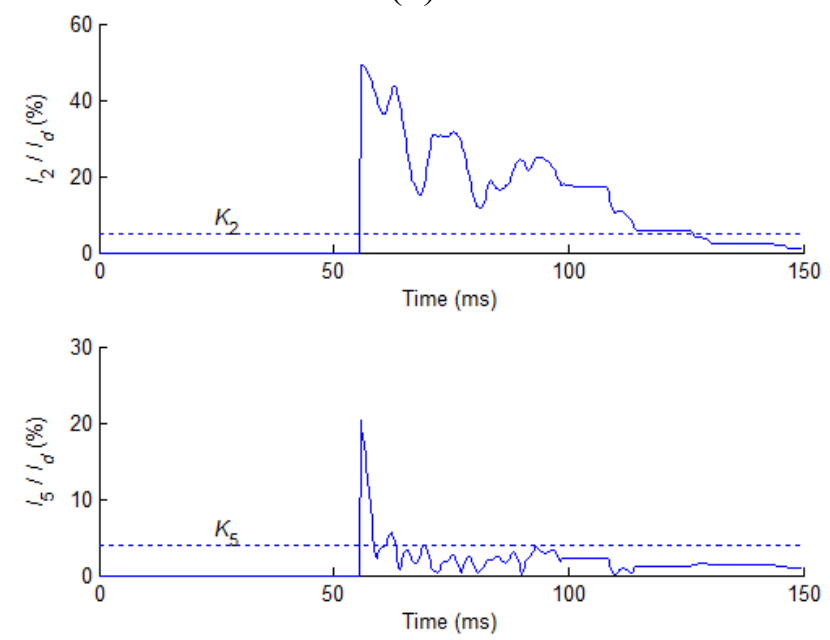

(c)

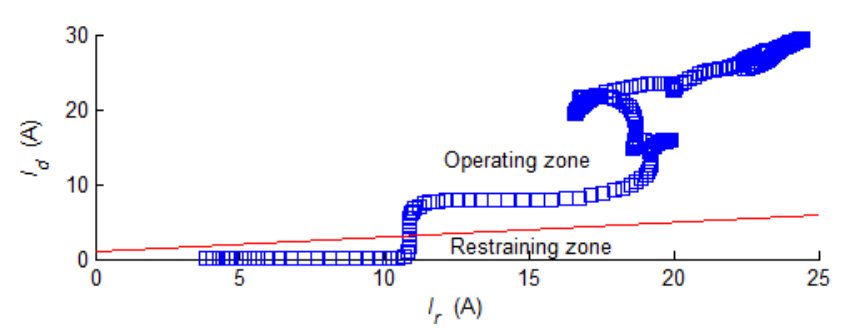

(b)
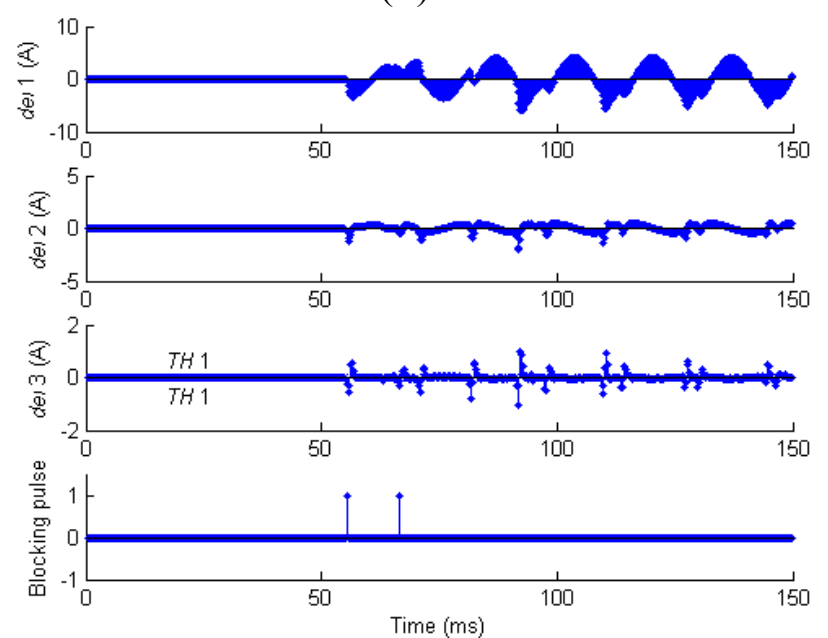

(d)

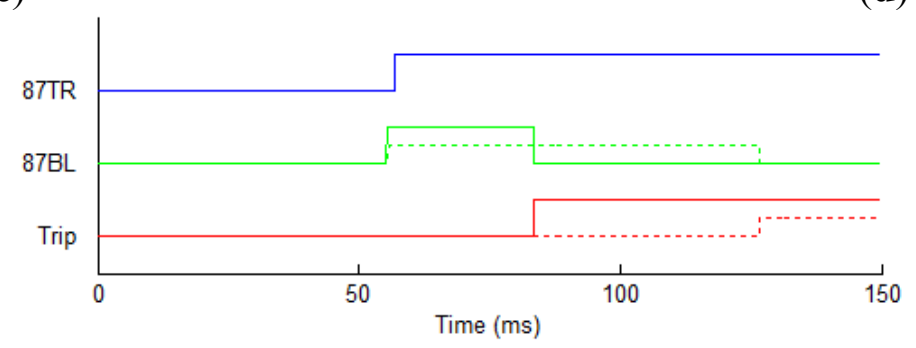

(e)

\section{Conclusions}

This paper described a current differential relay for transformer protection that operates in conjunction with a core saturation detection-based blocking scheme. The proposed saturation detection algorithm is based on the third-difference function of a differential current; it detects the start and end of each saturation period of the transformer core. At every point of inflection, which is the start or end of each saturation period, the blocking signal is issued and maintained for one cycle. In addition, a method to discriminate between transformer saturation and CT saturation is designed. 
The performance of the proposed blocking scheme is compared with that of the harmonic blocking scheme. The test results indicate that the proposed scheme successfully restrains the current differential relay like the conventional scheme for the magnetic inrush, over-excitation, and external faults with CT saturation; in addition, it significantly reduces the operating time delay of the relay in the case of an internal fault with $\mathrm{CT}$ saturation and an internal fault after an external fault. Moreover, the proposed saturation detection scheme successfully discriminates CT saturation from power transformer saturation.

\section{Acknowledgments}

This work was supported by the National Research Foundation of Korea (NRF) grant funded by the Korea government (MSIP) (2010-0028509).

\section{Author Contributions}

All the authors contributed to publish this paper. Byung Eun Lee, Sung Ho Won, and Yong Cheol Kang mainly proposed the algorithm of this paper. Byung Eun Lee has carried out the simulation tests and Yong Cheol Kang has checked the simulation result. Writing was done by Byung Eun Lee, Jinsik Lee, Sung Ho Won, Byongjun Lee, Peter A. Crossley, and Yong Cheol Kang. Final review was done by Byung Eun Lee, Jinsik Lee, Byongjun Lee, Peter A. Crossley, and Yong Cheol Kang.

\section{Conflicts of Interest}

The authors declare no conflict of interest.

\section{References}

1. Phadke, A.G.; Thorp, J.S. Computer Relaying for Power Systems; Research Studies Press: Hertfordshire, UK, 1988.

2. Hayward, C.D. Harmonic-current-restrained relays for transformer differential protection. Electr. Eng. 1941, 60, 377-382.

3. Mathews, C.W. An improved transformer differential relay. AIEE Trans. Part III 1954, 73, 645-650.

4. Sharp, R.L.; Glassburn, W.E. A transformer differential relay with second-harmonic restraint. Power Appar. Syst. Part III Trans. Am. Inst. Electr. Eng. 1958, 77, 913-918.

5. Einvall, C.H.; Linders, J.R. A three-phase differential relay for transformer protection. IEEE Trans. Power Appar. Syst. 1975, 94, 1971-1980.

6. Rockefeller, G.D. Fault protection with a digital computer. IEEE Trans. Power Appar. Syst. 1969, $88,438-462$.

7. Giuliante, A.; Clough, G. Advances in the design of differential protection for power transformers. In Proceedings of the Georgia Technical Protective Relaying Conference, Atlanta, GA, USA, 1-3 May 1991; pp. 1-12.

8. Harder, E.L.; Marter, W.E. Principles and practices of relaying in the United States. Trans. Am. Inst. Electr. Eng. 1948, 67, 1005-1023. 
9. Phadke, A.G.; Thorp, J.S. A new computer-based flux-restrained current-differential relay for power transformer protection. IEEE Trans. Power Deliv. 1983, 102, 3624-3629.

10. Inagaki, K.; Higaki, M.; Matsui, Y.; Kurita, K.; Suzuki, M.; Yoshida, K.; Maeda, T. Digital protection method for power transformers based on an equivalent circuit composed of inverse inductance. IEEE Trans. Power Deliv. 1988, 3, 1501-1510.

11. Sachdev, M.S.; Sidhu, T.S.; Wood, H.C. A digital relaying algorithm for detecting transformer winding faults. IEEE Trans. Power Deliv. 1989, 4, 1638-1648.

12. Kang, Y.C.; Lee, B.E.; Kang, S.H.; Crossley, P.A. Transformer protection based on the increment of the flux linkages. IEE Proc. Gener. Trans. Distr. 2004, 151, 548-554.

13. Bo, Z.; Weller, G.; Lomas, T. A new technique for transformer protection based on transient detection. IEEE Trans. Power Deliv. 2000, 15, 870-875.

14. Saleh, S.A.; Rahman, M.A. Real-time testing of a WPT-based protection algorithm for three-phase power transformers. IEEE Trans. Ind. Appl. 2005, 41, 1125-1132.

15. Saleh, S.A.; Scaplen, B.; Rahman, M.A. A new implementation method of wavelet-packet-transform differential protection for power transformers. IEEE Trans. Ind. Appl. 2011, 47, 1003-1012.

16. Tripathy, M.; Maheshwari, R.P.; Verma, H.K. Radial basis probabilistic neural network for differential protection of power transformer. IET Gener. Trans. Distr. 2008, 2, 43-52.

17. Tripathy, M.; Maheshwari, R.P.; Verma, H.K. Power transformer differential protection based on optimal probabilistic neural network. IEEE Trans. Power Deliv. 2010, 25, 102-112.

18. Oliveira, L.M.R.; Cardoso, A.J.M. Application of Park's power components to the differential protection of three-phase transformers. Electr. Power Syst. Res. 2012, 83, 203-211.

19. Oliveira, L.M.R.; Cardoso, A.J.M. Extended Park's vector approach-based differential protection of three-phase power transformers. IET Electr. Power Appl. 2012, 6, 463-472.

20. Alencar, R.J.N.; Bezerra, U.H.; Ferreira, A.M.D. A method to identify inrush currents in power transformers protection based on the differential current gradient. Electr. Power Syst. Res. 2014, $111,78-84$.

21. Kang, Y.C.; Won, S.H.; Kang, S.H.; Crossley, P.A. A transformer differential relay with core saturation detection algorithm. In Proceedings of the 8th IEE International Conference on Developments in Power System Protection, Amsterdam, The Netherlands, 5-8 April 2004; pp. 368-371.

22. Bastard, P.; Bertrand, P.; Meunier, M. A transformer model for winding fault studies. IEEE Trans. Power Deliv. 1994, 9, 690-699.

(C) 2014 by the authors; licensee MDPI, Basel, Switzerland. This article is an open access article distributed under the terms and conditions of the Creative Commons Attribution license (http://creativecommons.org/licenses/by/3.0/). 